\title{
Destruction of Be star disk by large scale magnetic fields
}

\author{
Asif ud-Doula ${ }^{1}$, Stanley Owocki ${ }^{2}$, Nathaniel (Dylan) Kee ${ }^{3}$ and \\ Michael Vanyo ${ }^{1}$ \\ ${ }^{1}$ Penn State Worthington Scranton, Dunmore, PA, USA, \\ email: asif@psu.edu \\ ${ }^{2}$ University of Delaware, Newark, DE, USA, \\ ${ }^{3}$ University of Tubingen, Tubingen, Germany
}

\begin{abstract}
Classical Be stars are rapidly rotating stars with circumstellar disks that come and go on time scale of years. Recent observational data strongly suggests that these stars lack the $10 \%$ incidence of global magnetic fields observed in other main-sequence B stars. Such an apparent lack of magnetic fields may indicate that Be disks are fundamentally incompatible with a significant large scale magnetic field. In this work, using numerical magnetohydrodynamics (MHD) simulations, we show that a dipole field of only $100 \mathrm{G}$ can lead to the quick disruption of a Be disk. Such a limit is in line with the observational upper limits for these objects.
\end{abstract}

Keywords. stars: emission-line, Be, stars: magnetic fields, stars: mass loss, (magnetohydrodynamics:) MHD

There is no unique definition of a Be star. The most common one, popularized by Collins (1987) defines a Be star as 'a non-supergiant B star whose spectrum has, or had at some time, one or more Balmer lines in emission'. Despite some problems with this broad definition, implication is that a Be star at some point of its life hosted a disk of circumstellar dense material. Material in such disks have been shown to be in Keplerian orbit and the host stars themselves have been found to be rapidly rotating. Recent extensive search of globally organized magnetic field in Be stars, especially by teams from MiMeS project, yielded null results, putting an upper limit of few tens $\mathrm{G}$ to such possible fields. In this work, we demonstrate that strong global magnetic field (dipolar) in excess of 50-100G are fundamentally incompatible with Keplerian disk in Be stars, at least partially explaining the lack of detectable magnetic fields in such objects.

We set up a numerical parameter study of disk-field interaction (along with weak radiatively driven wind) of a typical Be star. We assume, initially, a pure global dipole field anchored in the interior of the star, and a pre-existing Keplerian disk surrounding the star. Weak radiatively driven wind has very little dynamical effects on the disk, but nonetheless is kept for the sake consistency. For various models, we change only the strength of the magnetic field. We find that any field in access of $10 \mathrm{G}$ has significant dynamical effects on the disks. Our highest field strength model (100G) completely disrupts the Keplerian disk.

Our models do not account for rapid stellar rotation, and associated with it gravity darkening. All these could affect the threshold of our results, although we do not believe they would alter our basic conclusion that strong magnetic fields are incompatible with Keplerian disks in Be stars. 